\title{
Resection and Reconstruction of the Trachea for the Treatment of Upper Tracheal Stenosis: Tracheal Versus Non-Tracheal Intubation
}

\author{
Feng Li1, Jing Liang2 and Xiaogang Li1
}

\begin{abstract}
Tracheal stenosis may occur secondary to trauma, tumors, infections, and inflammatory and iatrogenic diseases. Patients with tracheal stenosis must be evaluated carefully and require comprehensive preoperative examination, including pulmonary function tests and radiographic studies. Two male patients (one 18 years of age and the other 46 years of age) with tracheal stenosis were scheduled for tracheal resection and reconstruction. They were given different modes of anesthesia (with and without intubation). Fiberoptic intubation was selected in the 18-year patient, and non-intubation was selected in 46-year patient. Invasive blood pressure monitoring was established prior to the induction of anesthesia. Both operations were successful without any untoward events. However, the choice of intubation resulted in prolonged stay and increased overall expenses. Therefore, it is suggested that non-tracheal intubation anesthesia should be used for resection of stenosis due to rapid postoperative recovery, shorten hospital stay, and lower cost. However, sufficient sedation and analgesia should be given and careful attention should be paid to patients.
\end{abstract}

Key Words: Trauma, Tracheal stenosis, Non-intubation anesthesia, Tracheal reconstruction.

\section{INTRODUCTION}

Tracheal stenosis is a well-recognised complication of trauma, iatrogenic causes, tumors, infections, and inflammatory diseases. ${ }^{1}$ Stenosis in the upper trachea is typically treated with tracheal resection and reconstruction via cervical incision under general anesthesia with tracheal intubation. However, tracheal intubation runs the risk of tracheal restenosis, especially in patients who need long-term mechanical ventilation after operation.

In this article, we discuss two cases of upper tracheal stenosis resection and reconstruction with different techniques of airway management (intubation and non-intubation) and compare the advantages and disadvantages.

\section{CASE REPORT}

Case 1: An 18-year patient presented with progressive dyspnea and accompanying cough for three months after treatment with tracheal intubation and mechanical ventilation because of organophosphorus pesticide poisoning. Computed tomography (CT) showed stenosis

\footnotetext{
1 Department of Anesthesiology, First Affiliated Hospital of Xi'an Jiaotong University, Xi'an 710061, China

2 Department of Radiotherapy, Shaanxi Provincial Tumor Hospital, Affiliated Hospital of Xi'an Jiaotong University, Xi'an 710061, China

Correspondence: Dr. Feng Li, Department of Anesthesiology, First Affiliated Hospital of Xi'an Jiaotong University, Xi'an 710061, China

E-mail: doctorlifeng@xjtu.edu.cn
}

Received: April 19, 2018; Accepted: July 10, 2018 in the upper trachea. The size of stenotic segment was $30 \mathrm{~mm} \times 6.5 \mathrm{~mm}$ (Figure 1). The stenosis led to pneumonia, shortness of breath, and worsening dyspnea. Resection of stenosis was performed to alleviate the respiratory symptoms.

Non-invasive blood pressure, $\mathrm{SpO}_{2}$, ECG, BIS were used for monitoring, and invasive blood pressure monitoring was established prior to induction. Two mg midazolam was given for sedation; propofol (2 - $2.5 \mathrm{mg} / \mathrm{kg}$ ), remifentanil (4 - $5 \mu \mathrm{g} / \mathrm{kg})$, succinylcholine $(1-2 \mathrm{mg} / \mathrm{kg}$ ) were used for rapid anesthesia induction. Fiberoptic intubation was selected for anesthesia induction, and 6.0 tracheal catheter for intubation. Moreover, $50 \%$ oxygen was delivered at a rate of $3 \mathrm{~L} / \mathrm{min}$. Intravenous anesthetics (propofol 3 - $4 \mathrm{mg} / \mathrm{kg} . \mathrm{h}$, remifentanil 0.1 - 0.2 $\mu \mathrm{g} / \mathrm{kg}$.min) and sevoflurane $(0.5 \%-1 \%)$ were provided. The patient was steady throughout the anesthesia.

The patient's neck was hyperextended during the operation. A $4 \mathrm{~cm}$ incision was made in the neck. The trachea was carefully dissociated to avoid any injury to the recurrent laryngeal nerve and thyroid. The resection length of the tracheal stenosis was $30 \mathrm{~mm}$. The cuff of the endotracheal catheter was placed under the stenosis, which prevented blood from entering the lungs. We reconstructed the tracheal stump by continuous suture using 3-0 Prolene sutures. Air leak test of trachea was done by using water, no leak was observed. After effective hemostasis, the cervical incision was closed. The operation lasted for $80 \mathrm{~min}$, and the intraoperative blood loss was $20 \mathrm{~mL}$. The patient remained stable throughout the operation. After the surgery, the patient was sent to the intensive care unit with assisted mechanical ventilation. The tracheal catheter was removed six hours later. Antibiotics and ambroxol were 
administered to alleviate the symptoms of pneumonia. The patient was discharged 1 week later, and after five years of follow-up, good recovery was achieved.

Case 2: A 46-year patient presented with progressive dyspnea, shortness of breath, chest tightness, and thick sputum for 20 days. The patient suffered from accident trauma and multiple fractures 40 days before admission; and then received fracture fixation and diaphragmatic repair in another hospital for 20 days. After the emergency operation, the patient was sent to intensive care unit with assisted mechanical ventilation. Enhanced CT showed a stenosis in the upper trachea, with a size of $17.5 \mathrm{~mm} \times 2.4 \mathrm{~mm}$ (Figure 2). Given that the dyspnea, shortness of breath, and chest tightness were gradually worsening, resection of tracheal stenosis was performed to alleviate the respiratory symptoms.

Non-invasive blood pressure, $\mathrm{SpO}_{2}, \mathrm{ECG}, \mathrm{BIS}$, and invasive blood pressure were used; $100 \%$ oxygen was

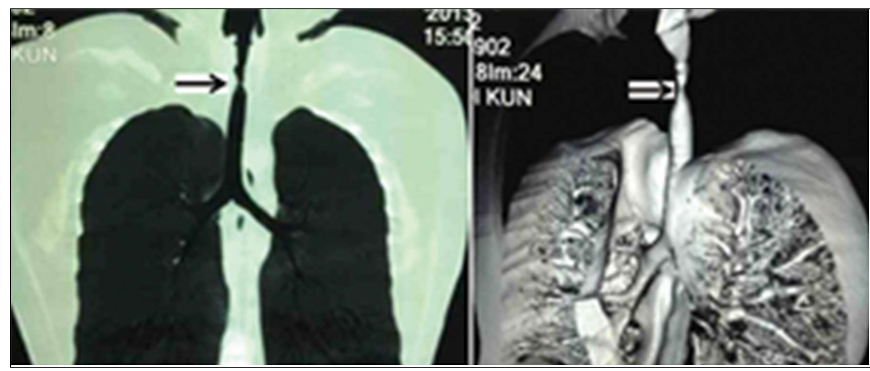

Figure 1: Chest CT of patient 1. The size of tracheal stenosis was $30 \mathrm{~mm} x$ $6.5 \mathrm{~mm}$.

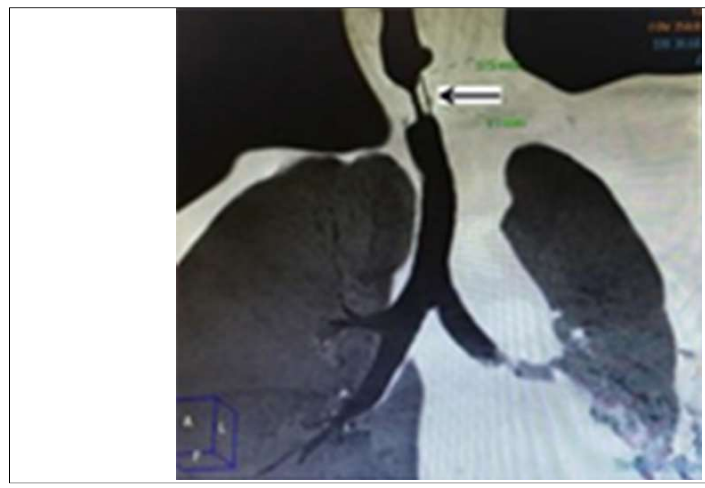

Figure 2: CT of patient 2. The size of tracheal stenosis was $17.5 \mathrm{~mm} \times 2.4 \mathrm{~mm}$.

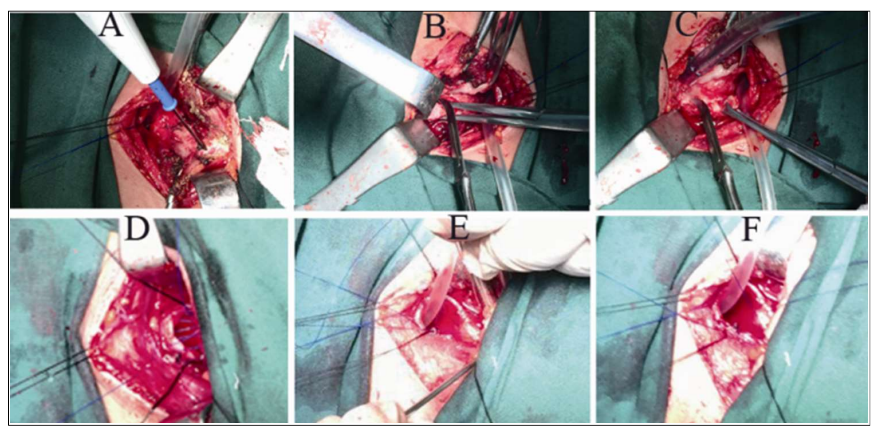

Figure 3 (A-F): Operation process for the second case delivered using a face mask at a rate of $5 \mathrm{~L} / \mathrm{min}$ after patient entering the operation room. One $\mathrm{mg}$ midazolam was given for sedation. Thirty minutes before the operation, the Intravenous infusion of dexmedetomidin $(0.8-2 \mu \mathrm{g} / \mathrm{kg} / \mathrm{h})$ was given, and bilateral superficial cervical plexus was blocked. Non-steroidal agents (parecoxib sodium $40 \mathrm{mg}$ for adults) and sufentanil $5-10 \mu \mathrm{g}(0.1-0.2 \mu \mathrm{g} / \mathrm{kg})$ were given before skin incision. One percent lidocaine was used for local anesthesia, especially around the trachea. Infusion of propofol (1-2 $\mathrm{mg} / \mathrm{kg} / \mathrm{h}$ ), maintaining the state of mild anesthesia, was done according to bispectral index (BIS) $(70$ - 80). After the trachea was completely disconnected, the patient's ventilation was greatly improved. Propofol $(0.5-1 \mathrm{mg} / \mathrm{kg})$ was given to maintain the value of the BIS at $60-70$ and then suck the phlegm.

The patient remained awake and was breathing spontaneously throughout the operation with mild sedation and analgesia. The depth of anesthesia was measured using the BIS. In addition, devices for tracheal intubation and mechanical ventilation were also prepared for emergency situation and to ensure the patient's safe. The patient was stable throughout the anesthesia.

The patient's neck was hyperextended during the operation, and a face mask was used. A $3 \mathrm{~cm}$ incision was made in the neck. The trachea was carefully dissociated to avoid any injury to the recurrent laryngeal nerve and thyroid. After tracheal incision, the symptoms of dyspnea were improved significantly. A catheter with oxygen was placed at the tracheal carina (Figures $3 \mathrm{E}$ and $\mathrm{F}$ ) to keep $\mathrm{SpO}_{2}>95 \%$. The tracheal stump was reconstructed by continuous suture using 4-0 Prolene sutures (Figures 3D-F). After no tracheal leak and active bleeding were found, the cervical incision was closed. The operation lasted for $90 \mathrm{~min}$, and the intraoperative blood loss was $10 \mathrm{~mL}$. The patient remained stable throughout the operation. One hour after the surgery, the patient was sent back to the ward. Six hours later, normal diet was resumed. The patient kept his mandible close to the sternum for reducing the tracheal tension after the surgery for 3 weeks, and he recovered earlier than did the case 1.

Postoperated CT examination showed that the pneumonia disappeared and the lungs re-expanded three days after the operation. The symptoms of dyspnea, shortness of breath, and chest tightness were significantly improved. No surgery-related complications (bleeding, tracheal re-stenosis, fever, pneumonia) were observed, and no antibiotics were required. On day 4, the patient was discharged. After one year of follow-up, no surgical complications were found.

\section{DISCUSSION}

Tracheal stenosis signifies a functional impairment, with peak expiratory flow rates changing from $100 \%$ in a 
normal trachea with a diameter of $2 \mathrm{~cm}$ to $30 \%$ in a trachea with a diameter of $5 \mathrm{~mm} .^{2}$ Tracheal stenosis causes difficulty in discharging sputum; if infected, causes pneumonia. Tracheal resection and reconstruction is the only way to treat this disease. Anesthesia is important for the operation to keep the vital sign stable.

Buckingham et al. first reported the use of thoracic epidural anesthesia to perform awake thoracic surgery. ${ }^{3}$ Surgical procedures using non-intubation anesthesia are more challenging to perform than those using intubation anesthesia; however, they greatly benefit patients as they recover sooner with less complications. Macchiarini et al. emphasised that non-intubated airway surgery not only avoids general anesthesia with intubation and mechanical ventilation, but also provides an ideal surgical field. Furthermore, the maintenance of spontaneous breathing makes airway reconstruction more anatomical and enables checking the movement of vocal cords during the surgical procedure. 4

It is important to note that non-tracheal intubation anesthesia should be avoided in patients with cardiocerebrovascular diseases, pulmonary insufficiency, and obesity. 5 In recent years, non-tracheal intubation surgery has been widely used to treat upper tracheal stenosis due to its advantage of accelerating postoperative recovery. 6 Non-intubation anesthesia for tracheal stenosis has distinct challenges and requires more cooperation of anesthesiologists and surgeons. During the operating procedure, endotracheal intubation, mechanical ventilation, and extracorporeal circulation devices (especially for anesthesiologist's lack of nonintubation experience) must be prepared for safety consideration.

The inadequacies of anesthesia of the second patient were the sedation and analgesia a little deficiency. The non-intubated patient felt a little pain during dissociation of the tissue around the trachea, especially the posterior wall of the trachea. The patient was choking cough when sucking phlegm (heart rate increased and blood pressure rose rapidly). Therefore, it is suggested that sufficient sedation and analgesia should be given. But it is important to pay attention to avoid respiratory depression. If the patient's breathing is completely inhibited and apnea occurs during the operation, trachea tube is instantly used to assist ventilation.

Non-tracheal intubation anesthesia has more advantages than tracheal intubation anesthesia for upper tracheal stenosis. Tracheal resection and reconstruction with spontaneous respiration via a neck incision is safe and has less complication. As anesthesiologists, we thought non-tracheal intubation anesthesia is an alternative method for the tracheal resection and reconstruction of upper tracheal stenosis, as it accelerates the recovery of patients.

The non-tracheal intubation anesthesia has more advantages than intubation anesthesia for the tracheal resection and reconstruction of upper tracheal stenosis. It enhances recovery after surgery, shortens hospital stay, and reduces the cost. For patient with tracheal stenosis, intubation anesthesia may increase the risk of re-stenosis. It can be well accepted by patients while sufficient sedation and analgesia are given. Moreover, careful attention should be paid to patients for retention of spontaneous breathing and maintaining circulation stability.

\section{REFERENCES}

1. Farzanegan R, Feizabadi M, Ghorbani F. An overview of tracheal stenosis research trends and hot topics. Arch Iran Med 2017; 20:598-607.

2. Daumerie G, Su S, Ochroch EA. Anesthesia for the patient with tracheal stenosis. Anesthesiol Clin 2010; 28:157-74.

3. Buckingham WW, Beatty AJ, Brasher CA, Ottosen P. The technique of administering epidural anesthesia in thoracic surgery. Dis Chest 1950; 17:561-8.

4. Macchiarini P, Rovira I, Ferrarello S. Awake upper airway surgery. Ann Thorac Surg 2010; 89:387-90.

5. Liu J, Li S, Shen J. Non-intubated resection and reconstruction of trachea for the treatment of a mass in the upper trachea. $J$ Thorac Dis 2016; 8:594-9.

6. Xu J, Yao Z, Li S, Chen L. A non-tracheal intubation (tubeless) anesthetic technique with spontaneous respiration for upper airway surgery. Clin Invest Med 2013; 36:E151-7. 